\title{
Estudio comparativo de la resistencia a la corrosión en juntas soldadas por fricción-agitación y por el proceso GMAW de aluminio AA1100
}

\author{
A comparative study of corrosion resistance in welded joints of aluminium \\ alloy AA1100 obtained by friction-stir and Gas Metal arc welding processes \\ Rafael J. Rodríguez ${ }^{1} \quad$ Carlos A. Caballis ${ }^{1} \quad$ María Mercedes Cely B. ${ }^{1} \quad$ Jimy Unfried-Silgado $^{2 *}$ \\ Recibido 24 de agosto de 2016, aceptado 3 de julio de 2017 \\ Received: August 24, 2016 Accepted: July 3, 2017
}

\begin{abstract}
RESUMEN
En este estudio se evaluó el comportamiento a la corrosión de juntas soldadas obtenidas mediante soldadura por fricción-agitación y por fusión al arco eléctrico de aleación de aluminio AA1100. Con el fin de comparar los efectos de ambos procesos sobre la resistencia a la corrosión, se realizaron pruebas en cámara de niebla salina y ensayos de polarización potenciodinámica, usando cloruro de sodio con base en las normas ASTM B117 y ASTM G5, respectivamente. Se analizaron la velocidad de corrosión, pérdida de masa y curvas de comportamiento potenciodinámico. Los análisis fueron complementados con estereología y con observación de las superficies ensayadas por medio de microscopia electrónica de barrido. Los resultados muestran que la corrosión fue localizada en ambos procesos de soldadura, siendo predominante en la región de soldadura para los procesos al arco. Se presentó para el proceso al arco mayores pérdidas de masa y velocidad de corrosión que para el proceso en estado sólido.
\end{abstract}

Palabras clave: Aleación de aluminio, soldadura, corrosión, ensayos potenciodinámicos.

\begin{abstract}
In this study was evaluated the corrosion behavior of welded joints obtained by electric arc and friction stir welding in AA1100 aluminum alloy with the aim to compare the effects of the process on the corrosion resistance. Tests were performed on salt spray chamber and potentiodynamic polarization test on welded joints based on ASTM B117 and ASTM G5 standards, respectively, using NaCl. The corrosion rate, mass loss behavior and potentiodynamic curves were analyzed. The analyzes were complemented by observation of tested surfaces with stereology and scanning electron microscopy. The results showed that localized corrosion was observed in both processes, which was stronger in the fusion region of welding arc processed samples. Furthermore, this last kind of samples showed higher mass loss and higher corrosion rate than the solid state processed samples.
\end{abstract}

Keywords: Aluminium alloys, welding, corrosion, potentiodynamic test.

\footnotetext{
1 Programa de Ingeniería Mecánica. Universidad Autónoma del Caribe. Grupo de Investigación en Materiales, Procesos y Tecnologías de Fabricación - IMTEF. Calle 90 No 46-112 Bl G. Barranquilla, Colombia.

2 Facultad de Ingeniería. Ingeniería Industrial. Universidad de la Costa. Grupo de Investigación en Productividad y Competitividad - PRODUCOM. Calle 58 No 55-66. Barranquilla, Colombia. E-mail: jimyunfried@ correo.unicordoba.edu.co

* Autor de correspondencia.
} 


\section{INTRODUCCIÓN}

El aluminio y sus aleaciones son los materiales metálicos no ferrosos más utilizados alrededor del mundo para la fabricación de estructuras civiles y mecánicas [1]. Estas aleaciones se caracterizan por su baja densidad, elevadas conductividades eléctrica y térmica, además de muy buena resistencia a la corrosión. Asimismo, gracias a su estructura cristalina cúbica centrada en las caras, el aluminio posee buena ductilidad. Entre las aleaciones de aluminio conformadas existen las tratables y las no tratables térmicamente [2]. La serie 1 xxx hace parte de las aleaciones no tratables térmicamente o endurecidas por deformación plástica.

Las aleaciones de aluminio de la serie $1 \mathrm{xxx}$ son denominadas también, aluminio comercialmente puro, pues solo contienen pequeñas adiciones de $\mathrm{Si}+\mathrm{Fe}<0,95 \%$ y $\mathrm{Cu}<0,20 \%$ en peso para dar resistencia mecánica al material sin propiciar el surgimiento de nuevas fases diferentes a la fase matriz FCC. Las aleaciones de la serie 1xxx, se suministran en dos condiciones: recocidas (-O) y endurecidas $(-\mathrm{H})$. Algunas de las principales propiedades mecánicas para ambas condiciones se presentan en la Tabla 1 [3].

Tabla 1. Propiedades de la aleación de aluminio AA1100 en diferentes condiciones.

\begin{tabular}{|l|c|c|}
\hline \multirow{2}{*}{ Propiedad } & \multicolumn{2}{c|}{ Condición de aleación } \\
\cline { 2 - 3 } & $-\mathbf{O}$ & $-\mathbf{H 1 4}$ \\
\hline $\mathrm{S}_{\mathrm{MAX}}(\mathrm{MPa})$ & $75-105$ & $110-145$ \\
\hline $\mathrm{S}_{\mathrm{Y}}(\mathrm{MPa})$ & $20-35$ & $95-117$ \\
\hline$\varepsilon(\%)$ & $25-40$ & $10-20$ \\
\hline Dureza (Brinell) & 23 & 32 \\
\hline
\end{tabular}

Los procesos de soldadura actualmente más usados para fabricación de juntas soldadas de aleaciones de aluminio son: soldadura al arco protegido con gas y electrodo consumible (GMAW - Gas Metal Arc Welding) y el proceso en estado sólido por fricción-agitación (FSW - Friction Stir Welding) [4].

El proceso de soldadura al arco protegido con gas y electrodo consumible (GMAW) es el proceso al arco más usado para unir aleaciones de aluminio. Este utiliza un alambre enrollado como electrodo consumible, el que es alimentado a una velocidad constante. Se genera un arco entre el metal base y el alambre, cuyo calor resultante funde este proporcionando la unión en la junta [5].

Este proceso emplea parámetros como: intensidad de corriente, tensión de corriente, velocidad de arrastre de hilo, velocidad de arrastre de pistola y caudal del gas. Luego de ejecutado el proceso de soldadura por GMAW se obtiene una junta, la que posee a lo largo de esta, zonas con características particulares, las cuales se representan en la Figura 1 [6].

El metal base (MB) es la zona no afectada por el calor del proceso. Esta zona conserva las propiedades mecánicas y microestructurales originales del material a unir. La zona afectada térmicamente (ZAT) es la zona donde ocurren cambios drásticos a nivel de la estructura (recristalización y crecimiento de grano) como producto de la influencia del calor generado por el proceso. En la zona fundida (ZF) se encuentra la unión metalúrgica producto de la fusión del material de aporte y parte del metal base. En esta última zona hay cambio de estado del material y solidificación con cambio de orientaciones y tamaño de grano diferentes al metal base [6].

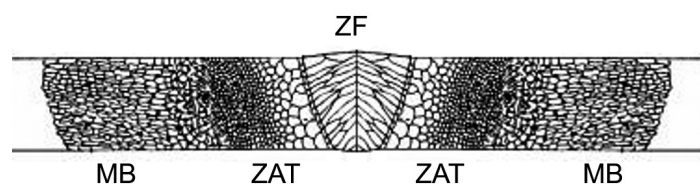

Figura 1. Vista transversal de la junta soldada por GMAW.

Por otro lado, el proceso de soldadura por fricción y agitación (SFA) viene ganando gran reputación en la unión de materiales de bajo punto de fusión [7]. SFA permite la unión de diversos materiales en estado sólido sin necesidad de utilizar consumibles para lograrlo. Este proceso usa una herramienta no consumible que gira y avanza para producir la unión sin necesidad de fusión. Entre sus principales ventajas están: bajos requerimientos de preparación de junta y ausencia de refuerzos. En este último caso se logra un menor nivel de tensiones residuales y como consecuencia un bajo grado de distorsiones, sin registrar defectos de solidificación, alta productividad con excelentes propiedades mecánicas de la unión 
soldada, las que son superiores muchas veces a las obtenidas por procesos convencionales al arco [8].

La Figura 2 muestra de forma esquemática el proceso de soldadura por fricción-agitación con sus principales parámetros de proceso, así como la sección transversal de una región típica de SFA. Este proceso emplea parámetros como: velocidad de avance (Vs), velocidad de rotación $(\mathrm{Vw})$ y fuerza axial $(\mathrm{Fz})$.

El calentamiento provocado por la fricción, producto de la velocidad de rotación $(\mathrm{Vw})$ junto con el desplazamiento de la herramienta $(\mathrm{V} s)$ hace que el material fluya creando una unión permanente entre ambas piezas. En SFA se suministra una fuerza en el sentido axial, perpendicular al plano de soldadura para mantener el flujo de material encauzado en la junta [8].

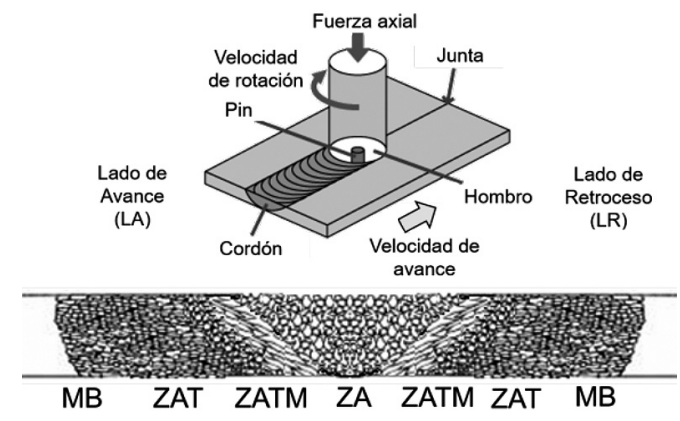

Figura 2. Proceso de soldadura por fricción y agitación.

Las regiones de soldadura del proceso SFA son ilustradas en la Figura 2. El metal base (MB) es la zona no afectada por el proceso, conservando las propiedades mecánicas y microestructurales originales. En la zona afectada térmicamente (ZAT), ocurren cambios a nivel microestructural (recristalización estática) producto de la influencia del calor generado por la fricción de la herramienta con el material. La zona afectada termomecánicamente (ZATM) es afectada por el calor producto del proceso y por la agitación del material. En esta zona los cambios estructurales son muy notorios en relación a la ZAT, observándose un flujo dinámico de material (recristalización dinámica) en una orientación distinta a las zonas MB y ZAT. Finalmente, la zona agitada (ZA) es donde se observa agitación severa del material producto del giro de la herramienta y el calor friccional.
Los cambios microestructurales en esta zona son los más grandes en comparación con las demás zonas. En la zona agitada la estructura del material está completamente recristalizada (producto de la recristalización dinámica severa) formando granos finos equiaxiales [9]. Las soldaduras de aluminio AA1100 no generan segundas fases, solo modificación del tamaño y morfología del grano en las diferentes zonas de soldadura [10].

El aluminio posee buena resistencia a la corrosión atmosférica, incluso a temperaturas relativamente altas y en diversos medios acuosos. Este material es muy activo en la serie electroquímica, pero se pasiva simplemente con la exposición al agua o al aire. La película pasiva está compuesta por óxido de aluminio, con un espesor aproximado entre $20 \AA$ a $100 \AA$ [11].

En general, los metales puros y comercialmente puros son mucho más resistentes a la corrosión que sus respectivas aleaciones. Las aleaciones de aluminio presentan buena resistencia a la corrosión en comparación a otros metales, debido a que el aluminio hace parte del grupo de metales con propiedades pasivantes, presentando baja resistencia al agua de mar [12]. Entre los factores más influyentes sobre la agresividad de la picadura en el aluminio están, el tipo de medio al que se está exponiendo, la temperatura en que se efectúa el ataque, la concentración y pH de la solución en la que se expone el metal y la superficie que se está evaluando en el metal [10, 12].

El comportamiento a la corrosión de las aleaciones de aluminio ha sido estudiado por diversos autores. Ezuber y colaboradores [13] expusieron aleaciones de aluminio AA5083 y AA1100 en agua de mar, con el fin de conocer su respuesta a la corrosión y poder generar un paralelo entre ambos comportamientos. Las curvas potenciodinámicas revelaron que las aleaciones de aluminio tipo AA1100 y AA5083 son menos vulnerables a la corrosión en agua de mar a temperatura ambiente, con mejor resistencia a la corrosión por parte de la aleación AA1100. Se revela un comportamiento más pasivante sobre la aleación AA1100 en ambas temperaturas de evaluación, siendo mayor la pasivación a $60^{\circ} \mathrm{C}$. Los resultados mostraron comportamientos anódicos, pero con buena participación pasivante por parte de ambas aleaciones antes de iniciar la picadura. 
Soltis y colaboradores [14] ejecutaron un experimento de polarización sobre un aluminio comercialmente puro AA1100, el que se expuso a solución salina de $\mathrm{NaCl}$ a diferentes concentraciones y a temperaturas que oscilaron entre $1{ }^{\circ} \mathrm{C}$ y $70{ }^{\circ} \mathrm{C}$, con el fin de establecer las concentraciones y temperaturas de mayor susceptibilidad a la corrosión. La incidencia de la corrosión fue proporcional a la disminución de la temperatura de la solución, para la solución de $0,5 \mathrm{~mol} \times \mathrm{dm}^{-3}$. Se reportó mejor respuesta a la corrosión en temperaturas altas con un óptimo en $70{ }^{\circ} \mathrm{C}$. La intensidad de picadura disminuyó a medida que se incrementa la temperatura.

Rajakumar y Balasubramanian [15] realizaron un estudio en el que expusieron juntas soldadas por SFA de AA1100 con diferentes parámetros en una solución de $\mathrm{NaCl}$ al 3,5\%-vol, para determinar la respuesta a la corrosión a diferentes velocidades de rotación y generar parámetros optimizados. Los resultados mostraron que todos los parámetros de proceso tienen efecto significativo en la velocidad de corrosión de la junta soldada. La velocidad de corrosión fue mínima cuando la velocidad de rotación estuvo en nivel medio, mientras que, a menor velocidad de soldadura y mayor velocidad de rotación, la tasa de corrosión fue máxima. La velocidad de corrosión más baja se obtuvo cuando todos los parámetros de SFA estaban en nivel medio y los resultados indican que a medida que la velocidad de rotación aumenta, la velocidad de corrosión disminuye. El objetivo de este artículo es evaluar el comportamiento a la corrosión de juntas soldadas obtenidas mediante soldadura por fricción y agitación y por fusión al arco eléctrico de aleación de aluminio AA1100 con el fin de comparar los efectos del proceso sobre la resistencia a la corrosión para ambos procesos.

\section{PROCEDIMIENTO EXPERIMENTAL}

\section{Materiales}

Placas de aleación de aluminio AA1100 de 100×50×6 $\mathrm{mm}^{3}$ de espesor fueron usadas como metal base. La composición química y las propiedades del metal base son mostradas en la Tabla 2. Se tuvo especial cuidado con la trazabilidad de la orientación de laminado durante todo el proceso de evaluación para mantenerla perpendicular a la dirección de soldadura.
Tabla 2. Composición química y propiedades de la aleación AA1100 usada.

\begin{tabular}{|l|c|c|}
\hline \multicolumn{1}{|c|}{ Ítem } & Valor & $\begin{array}{c}\text { Composición } \\
\text { química (\%-peso) }\end{array}$ \\
\hline $\mathrm{S}_{\mathrm{MAX}}(\mathrm{MPa})$ & 94 & $>0,10(\mathrm{Si}+\mathrm{Fe})$ \\
\hline $\mathrm{S}_{\mathrm{Y}}(\mathrm{MPa})$ & 75 & $\mathrm{Cu} \sim 0,10 \%$ \\
\hline$\varepsilon(\%)$ & 34 & $\mathrm{Al}($ Balance $)$ \\
\hline Dureza (Brinell) & 40 & \\
\hline
\end{tabular}

\section{Obtención de juntas soldadas}

Las juntas soldadas obtenidas por el proceso GMAW fueron realizadas usando la geometría de junta mostrada en la Figura 3 (a). Se usaron los siguientes parámetros: corriente entre 160-190A, voltaje entre $23-26 \mathrm{~V}$, velocidad de alimentación del alambre entre 6,1 a 7,2 m. $\mathrm{min}^{-1}$ y una velocidad de soldadura entre $330-450 \mathrm{~mm} \cdot \mathrm{min}^{-1}$. Fue usado un equipo de soldadura Lincoln Electric ${ }^{\circledR}$ Weld 260 D, como gas de protección la mezcla de argón $80 \%$ $\mathrm{He}, 19 \% \mathrm{Ar}, 1 \% \mathrm{O}_{2}$, con un caudal de 5,5 $1 . \mathrm{min}^{-1}$ y un electrodo ER4043 de $\varnothing 1,2 \mathrm{~mm}$.
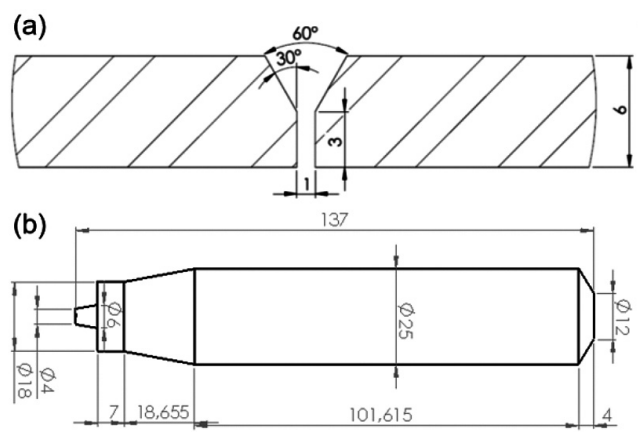

Figura 3. (a) Detalle de la junta utilizada para el proceso GMAW. (b) Herramienta usada para la aplicación del proceso SFA.

Las soldaduras obtenidas por el proceso SFA, fueron realizadas con herramienta de hombro plano y pin cónico roscado mostrada en la Figura 3 (b). La junta fue sin preparación y a tope. Se usaron los siguientes parámetros: velocidad de rotación entre 900 - 1.200 $\mathrm{rpm}$, velocidad de soldadura entre $90-120 \mathrm{~mm} \cdot \mathrm{min}^{-1}$ y como equipo de soldadura una fresadora marca Kondor ${ }^{\circledR}$ de 3,2 hp adaptada para tal fin.

\section{Preparación de probetas}

Las probetas para ensayos de corrosión en la cámara de niebla salina fueron obtenidas a partir de los 
cupones de soldadura, en la región estable del cordón (centro de la soldadura), en forma de placas con dimensiones $6 \times 55 \times 100 \mathrm{~mm}$. Estas placas fueron preparadas según la norma ASTM B117 [16], la que exige unas condiciones mínimas, tales como: limpieza de superficie, remoción de grasas y otras sustancias. Lavado con agua tibia y detergente con $\mathrm{pH}$ neutro. Luego se realiza el lijado de poros y hendiduras para evitar acumulación de sal. El pulido de la superficie se hace hasta alcanzar uniformidad (lija con granulometría 600). La Figura 4 muestra el aspecto de las probetas luego de la preparación. Se ensayaron tres muestras de SFA, dos de GMAW y dos de MB.

\begin{tabular}{|c|c|c|}
\hline \multirow{2}{*}{ Origen } & \multicolumn{2}{|c|}{ Estado de la superficie } \\
\cline { 2 - 3 } MB & Sin preparar & Preparadas \\
\hline \multirow{2}{*}{ GMAW } & & \\
\hline SFA & & \\
\hline
\end{tabular}

Figura 4. Aspecto de cuerpos de prueba para ensayo en cámara de niebla salina.

Las probetas para ensayos potenciodinámicos se prepararon según la norma ASTM G1 y G3 [1718] y se extrajeron de las soldaduras desde las localizaciones mostradas en las Figuras 5 y 6 .

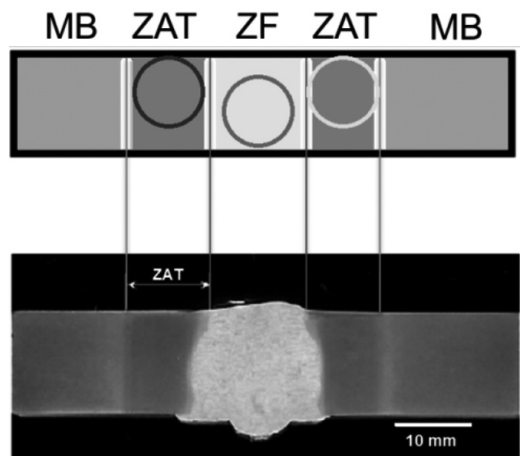

Figura 5. Localización de las probetas en la junta de GMAW.

La norma exige condiciones mínimas de las probetas tales como: limpieza de superficie, remoción de porosidades e imperfecciones, y pulido de la superficie, a fin de captar las mejores evidencias de corrosión. Se redujeron poros e imperfecciones de la superficie usando papel de lija con granulometría 600. Se extrajeron un total de 14 probetas para este ensayo.

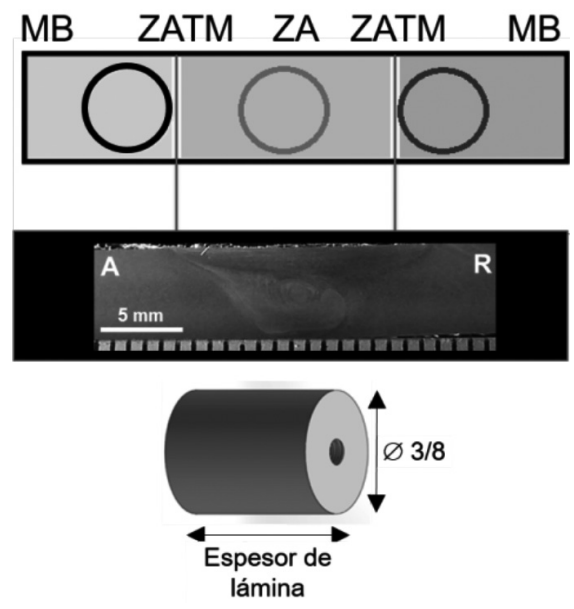

Figura 6. Localización de las probetas en la junta de SFA. Inferior, aspecto de la probeta.

\section{Ensayos de corrosión}

Luego que las probetas fueron obtenidas a partir de los cupones de soldadura, las mismas fueron sometidas a los ensayos de cámara de niebla salina y polarización potenciodinámica.

\section{Ensayos en cámara de niebla salina}

El ensayo en cámara de niebla salina fue llevado a cabo en una cámara Angelantoni ${ }^{\circledR}$ Industrie S.P.A. modelo DCTC 1200P. Las condiciones del ensayo fueron las siguientes: Niebla salina al $5 \% \mathrm{NaCl}$, con $\mathrm{pH}$ de $7 \pm 1$ y temperatura de $35^{\circ} \mathrm{C} \pm 2{ }^{\circ} \mathrm{C}$, como lo indica la norma ASTM B117-11. Los ensayos se ejecutaron durante 168 horas y para determinar el avance de corrosión las muestras fueron removidas en el gabinete cada 48 y 72 horas. Se realizó registro fotográfico y se calculó la pérdida de masa y tasa de corrosión para cada una de las probetas.

\section{Ensayos de polarización potenciodinámicas}

Para estos ensayos se utilizó un PotenciostatoGalvanostato marca Solartron Analytical ${ }^{\circledR}$. El software ModuLab ${ }^{\circledR}$ fue usado para la transducción y análisis de datos, en especial, para la tasa de corrosión. Se utilizó una solución de $\mathrm{NaCl}$ al 5\%. 
Los ensayos fueron realizados usando potenciales con valores entre -300 y $1.508,6 \mathrm{mV}$, con electrodo de calomel saturado (SCE) y velocidad de barrido de $1 \mathrm{mV} / \mathrm{min}$.

\section{Caracterización y análisis}

Las superficies de las muestras obtenidas en el ensayo de cámara de niebla salina fueron exploradas mediante una cámara digital de 13 megapíxeles acoplada a un celular que fue empotrado en un dispositivo especial de sujeción y debidamente calibrado con ayuda de patrones de microscopia óptica. Fue utilizada una cuadrícula superpuesta de 100 cuadros, cada uno de $5 \times 2,5 \mathrm{~mm}^{2}$, para cada imagen captada con la cámara digital. Con estas imágenes se realizó estereografía con el fin de determinar el porcentaje de corrosión y tipo de corrosión presente en cada una de las probetas ensayadas.

El análisis de la superficie en muestras sometidas a ensayos de polarización potenciodinámicas fue realizado en un microscopio electrónico de barrido JEOL® JSM-7100F, usando una energía de haz entre $10 \mathrm{kV}$ a $25 \mathrm{kV}$ y el modo de electrones secundarios, el cual fue usado para búsqueda de picaduras y clústeres de picaduras.

\section{RESULTADOS Y DISCUSIÓN}

\section{Ensayos en cámara de niebla salina}

El análisis de corrosión se determinó teniendo como referencia al material base. La Figura 7 muestra el análisis con la cuadricula de las superficies de las probetas ensayadas. Se evidencia un comportamiento de corrosión uniforme en el material base, mientras que probetas procesadas por SFA y GMAW muestran evidencia tanto de corrosión uniforme como localizada.
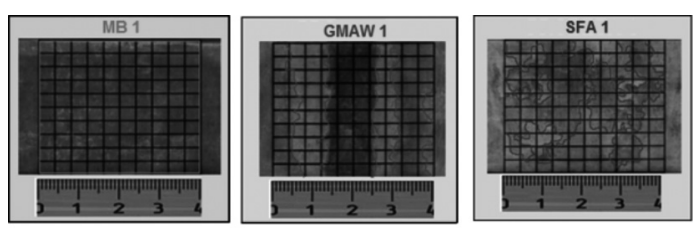

Figura 7. Análisis de la superficie de probetas MB, SFA y GMAW ensayadas en cámara de niebla salina.

La Figura 8 muestra los porcentajes de corrosión localizada y corrosión uniforme para cada probeta estimando un error del 5\%. Las probetas procesadas con SFA presentaron hasta 5 veces más corrosión localizada que el material base, mientras que probetas procesadas con GMAW mostraron hasta 8 veces más corrosión localizada comparada con el material base.

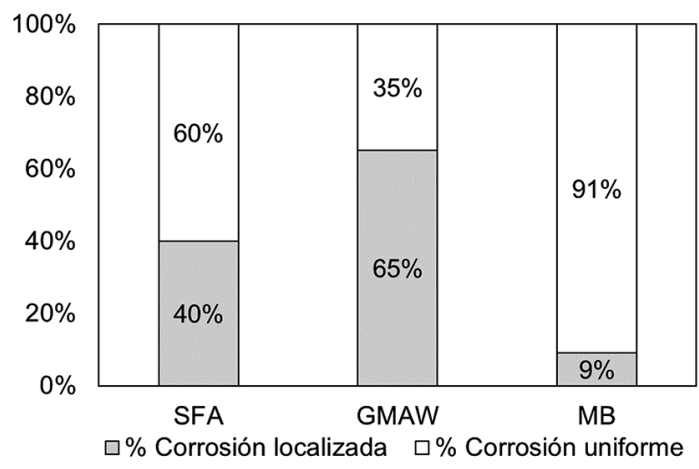

Figura 8. Porcentajes de corrosión localizada y corrosión uniforme sobre las probetas MB, SFA y GMAW.

La Figura 9 muestra las zonas de corrosión en las juntas soldadas. Se logra observar en las soldaduras obtenidas por GMAW (Figura 9 a) que predomina la corrosión localizada sobre la zona fundida (cordón) mientras que en la ZAT se tiene una buena respuesta a la corrosión. Por otra parte, en las juntas soldadas por SFA (Figura 9 b) se observa predominio de corrosión localizada en la región adyacente a la zona agitada y entre la zona agitada y el material base.

La Figura 10 muestra la pérdida de masa de las muestras sometidas a ensayo en cámara de niebla salina. Se evidencia mayor pérdida de masa en las probetas obtenidas con el proceso GMAW. Las probetas procesadas con SFA obtuvieron una pérdida de masa hasta 1,7 veces mayor con respecto al material base, en tanto que, las muestras procesadas con GMAW presentaron pérdida de masa hasta 2,3 veces mayor con respecto al material base. Con los datos de la pérdida de masa y la ecuación (1) fue calculada la tasa de corrosión por pérdida de masa para cada caso.

$$
V_{\text {corr }}=\frac{87,6 \mathrm{~W}}{A \times \rho \times t}
$$

Dónde $v_{c o r r}$ es la tasa de corrosión; $W$ es la pérdida de masa; $A$ el área de la probeta; $\rho$ es la densidad del material y $t$ el tiempo de exposición. 

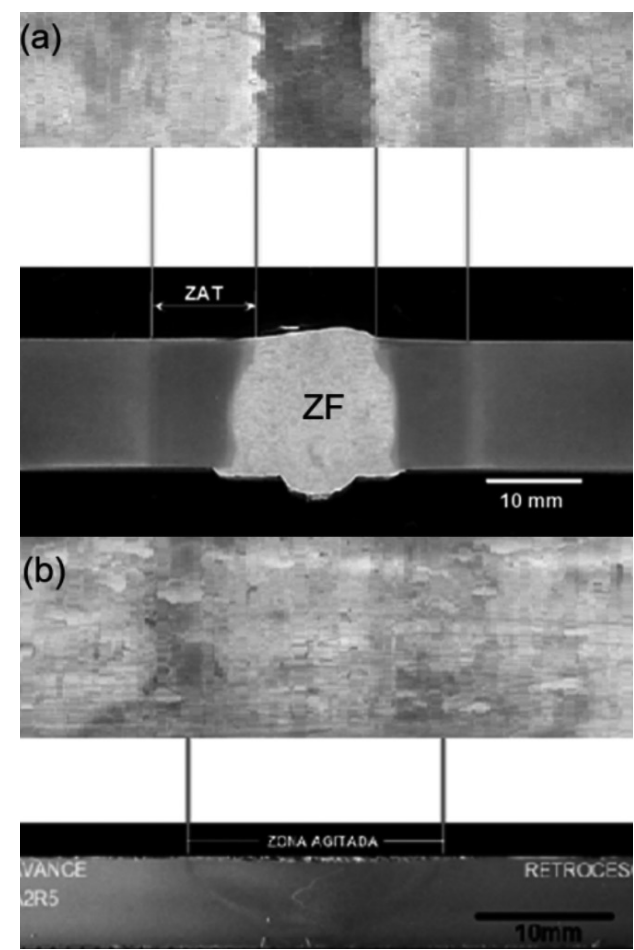

Figura 9. Vista global de las superficies de las probetas soldadas expuestas a cámara de niebla salina. (a) Proceso GMAW. (b) Proceso SFA.

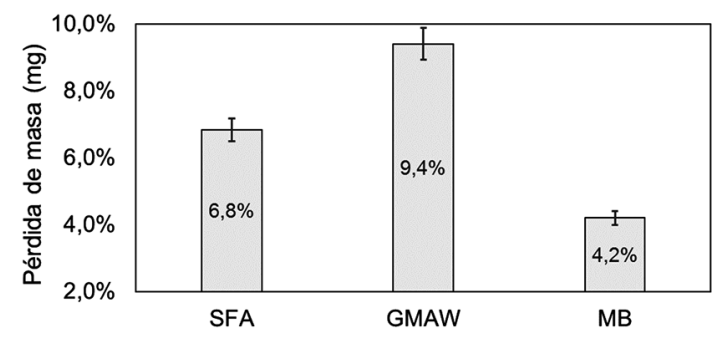

Figura 10. Pérdida de masa de MB y probetas procesadas por SFA y GMAW.

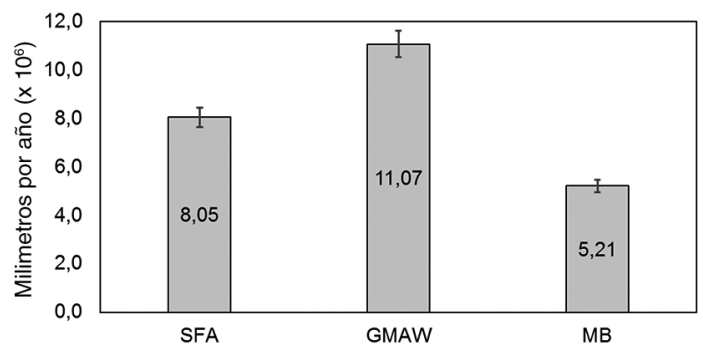

Figura 11. Tasa de Corrosión por pérdida de masa en mm/año.
La Figura 11 muestra la tasa de corrosión para cada caso. Las probetas con mayor tasa de corrosión fueron las procesadas por GMAW en comparación con las de SFA y el metal base.

\section{Pruebas de resistencia a la corrosión}

Las Figuras 12 y 13 muestran las curvas potenciodinámicas para probetas procesadas con GMAW y SFA, respectivamente. El proceso se efectuó mediante la agrupación de curvas correspondientes a las zonas evaluadas en cada caso, comparándolas con la curva de material base. El material base se comportó de forma anódica y catódica, pero sin variar sus características de respuesta a la corrosión natural, con presencia de pasivación y transpasivación en las curvas [13-14].

En la Figura 12 se analiza la curva de polarización de una junta soldada procesada por GMAW. Se observa que el potencial de la zona ZAT2 es más alto al compararlo con la zona ZAT1 y la zona fundida ZF. En general, se observa un menor potencial de corrosión en la ZAT y mayor potencial de corrosión en la zona fundida con comportamientos anódicos diferentes al final del tramo anódico. Se observa notable pasivación en la ZAT y zona del cordón ZF (por aumentar las densidades de corriente). Todas las curvas de polarización correspondientes al proceso GMAW presentaron potenciales más bajos comparados al material base. $[12,14]$.

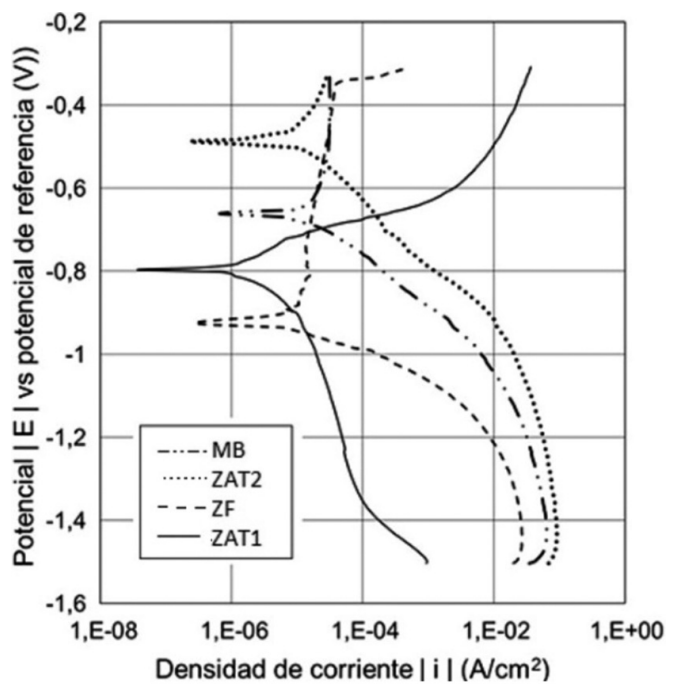

Figura 12. Curvas de polarización de muestras de aluminio AA110 soldadas por proceso GMAW. (ZAT1, ZAT2, ZF, MB). 
Por otro lado, el efecto de los parámetros de proceso sobre el comportamiento a la corrosión de las juntas soldadas por el proceso SFA es significativo, especialmente en el caso de la combinación de parámetros de velocidad de rotación alta (entre 900 a $1200 \mathrm{rpm}$ ) y velocidad de avance o de soldadura baja (entre 90 a $120 \mathrm{~mm} \cdot \mathrm{min}^{-1}$ ), propiciando tendencia a la corrosión, como lo observado por algunos autores [15].

La Figura 13 muestra un comportamiento muy uniforme de las zonas de estudio con respecto a la resistencia a la corrosión, observándose pequeñas variaciones en los potenciales de corrosión. Los resultados muestran que la zona agitada de SFA presenta un potencial de corrosión muy similar al de la zona de avance mientras que la zona de retroceso muestra un potencial levemente menor al de la zona agitada; sin embargo, el comportamiento de las curvas de polarización muestra que la zona de avance es la zona con mayor tendencia a la corrosión como se muestra en la Tabla 3. Este comportamiento se debe posiblemente al mayor calentamiento producido por el efecto aditivo de los vectores de velocidad tangencial del borde de la herramienta y la velocidad de avance de la misma. Este mayor calentamiento produce ligeros cambios en el nivel de recristalización de la zona de avance con relación a las zonas de retroceso y de agitación [19]. Por otro lado, la ausencia de segundas fases debido al tipo de aleación estudiada, puede explicar porque hay exiguas diferencias en el comportamiento a la corrosión de las diferentes zonas de soldadura para el proceso SFA aquí estudiado [19-20].

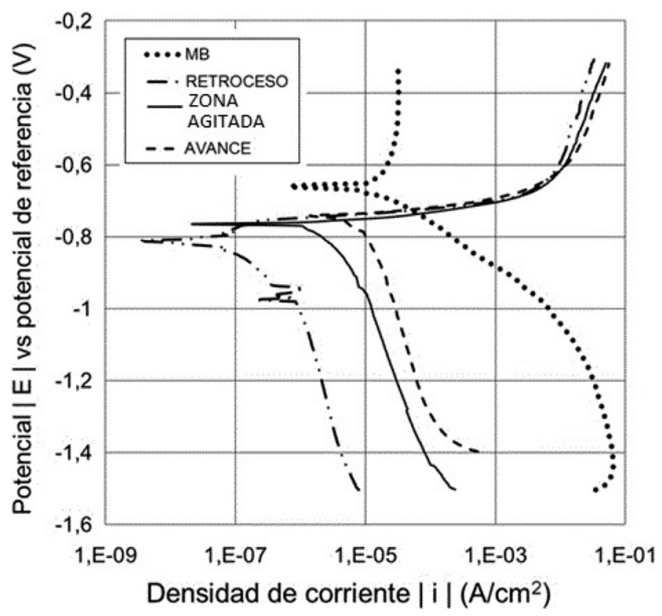

Figura 13. Curvas de polarización de muestras de aluminio AA1100 soldadas por proceso SFA.
Los potenciales de corrosión y de picadura son mostrados en la Tabla 3, identificando el potencial de corrosión, el que indica el paso de comportamiento catódico a un comportamiento anódico por parte del material, sin importar los comportamientos obtenidos en las fases anódicas y catódicas. La resistencia a la corrosión por picadura fue menos evidente en el proceso SFA debido al refinamiento de la estructura inherente a este proceso [19].

Tabla 3. Potenciales de corrosión para cada zona extraída.

\begin{tabular}{|l|l|c|c|}
\hline $\begin{array}{c}\text { Proceso de } \\
\text { soldadura }\end{array}$ & \multicolumn{1}{|c|}{$\begin{array}{c}\text { Zonas } \\
\text { extraídas }\end{array}$} & $\begin{array}{c}\text { Potencial de } \\
\text { corrosión } \\
\mathbf{( m V )}\end{array}$ & $\begin{array}{c}\text { Potencial de } \\
\text { picadura } \\
\mathbf{( m V )}\end{array}$ \\
\hline MB & MB & $-678,2$ & -387 \\
\hline \multirow{3}{*}{ SFA } & Avance & $-734,3$ & - \\
\cline { 2 - 4 } & Zona agitada & $-755,7$ & - \\
\cline { 2 - 4 } & Retroceso & $-805,4$ & - \\
\hline \multirow{3}{*}{ GMAW } & ZAT1 & $-801,4$ & - \\
\cline { 2 - 4 } & Cordón & $-933,2$ & -374 \\
\cline { 2 - 4 } & ZAT2 & $-483,2$ & - \\
\hline
\end{tabular}

La Figura 14 muestra la tasa de corrosión para cada una de las zonas. Los valores correspondientes a la tasa de corrosión recopilada en los ensayos en potenciostato, muestran que en general, las zonas de las probetas procesadas por SFA tienen diferencias significativas entre la zona de retroceso y la zona de avance. Las zonas de avance en SFA presentan mayores tasas de corrosión que las zonas de retroceso, lo que indica que las primeras parecen ser más vulnerables a la corrosión, mientras que las zonas de retroceso presentan mejor comportamiento a la corrosión. Por otra parte, la zona agitada de SFA presenta menor tasa de corrosión en

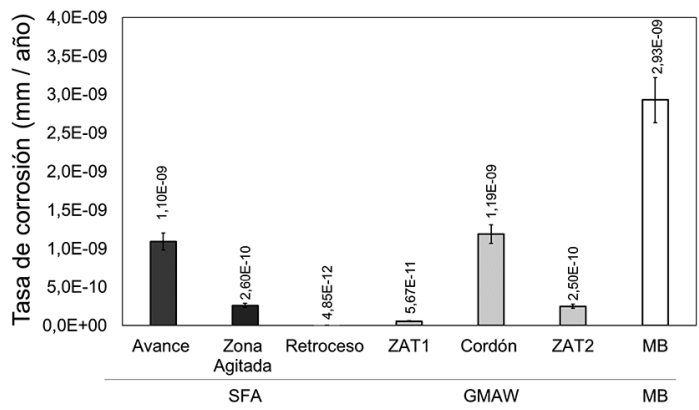

Figura 14. Tasa de corrosión para cada una de las zonas. 
comparación a la zona del cordón del proceso GTAW, mientras que la ZAT en este mismo proceso presenta comportamiento similar. La alta refinación del tamaño de grano en la zona agitada, produce un mayor número de límites de grano, debido a la recristalización en el proceso SFA [15,19], contrastada con las estructuras de solidificación columnares de mayor tamaño en el proceso GMAW [5], pueden ser explicaciones plausibles para el comportamiento de la corrosión observado.

Finalmente, la Figura 15 muestra imágenes de microscopia electrónica en igual magnificación de las picaduras observadas en las zonas de fusión (Figura 15 a) para el proceso GMAW y en la zona agitada (Figura 15 b) para el proceso SFA. Para las probetas procesadas con GMAW la zona fundida presenta corrosión localizada más alta en comparación con la ZAT y el metal base, principalmente, debido a la estructura de solidificación propia de esa zona. Se observa en la región de la ZAT un buen comportamiento a la corrosión, debido a la

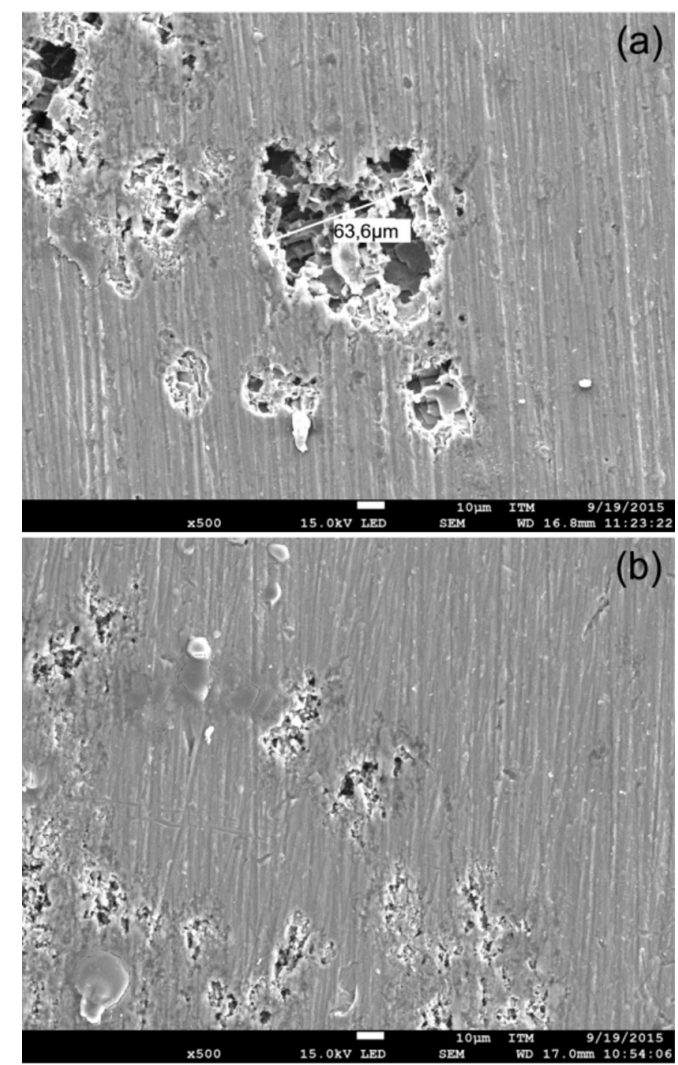

Figura 15. Aspecto de picaduras en las regiones de (a) zona de fusión o cordón para GMAW1 y (b) zona agitada para SFA3. recristalización, producto de la combinación entre la deformación previa del material soldado y la rápida tasa de enfriamiento de la probeta que genera zonas con grano fino y otras con crecimiento excesivo del grano [13-15].

Para los ensayos en las probetas obtenidas por el proceso SFA, se observaron picaduras relativamente pequeñas $(<10 \mu \mathrm{m})$ con una distribución muy dispersa. Las características de la corrosión localizada fueron más evidentes sobre la zona de retroceso, probablemente debido a que son zonas más frías en relación a las zonas más calientes o de avance [15]; además, en la zona de retroceso hay flujo de material hacia la parte inferior de la soldadura sin acumulación en esta zona, lo que podría explicar los resultados en los ensayos de potenciostato en la zona de retroceso. Los resultados obtenidos fueron similares a los observados en la literatura [13-15].

\section{CONCLUSIONES}

De acuerdo con los resultados obtenidos en esta investigación se puede concluir que la corrosión localizada predomina con mayor intensidad en las juntas soldadas por GMAW. La corrosión localizada en juntas soldadas por SFA se presentó en la región adyacente a la zona agitada siendo más evidente en la región de avance. La mayor pérdida de masa se registró en las juntas soldadas por GMAW con un promedio de 9,4 mg, mientras que las juntas SFA fue de $6,83 \mathrm{mg}$. La velocidad de corrosión por pérdida de masa en mm/año fue superior en juntas soldadas por GMAW. Los ensayos en potenciostato revelaron que la junta soldada que presentó en todas sus zonas comportamientos pasivantes representativos fue la SFA. La tasa de corrosión para los ensayos realizados en potenciostato presentaron diferencias marcadas para las 3 zonas en las probetas procesadas por SFA, mientras que en las probetas procesadas por GMAW presentan comportamientos similares en la ZAT, con incremento de la tasa de corrosión en la zona del cordón. La tasa de corrosión más baja para las zonas evaluadas en el proceso SFA fue para la zona de avance.

\section{AGRADECIMIENTOS}

Los autores de este trabajo agradecen el apoyo financiero de la Universidad Autónoma del Caribe 
por medio del proyecto interno CONV-I-004-P012. Igualmente, los autores agradecen al Dr. Juan F. Santa del Instituto Tecnológico Metropolitano (ITM) por su invaluable colaboración con las imágenes de microscopia y a la empresa ACESCO S.A. por el uso del equipo de cámara de niebla salina.

\section{REFERENCIAS}

[1] J.R. Davis. "Aluminum and Aluminum Alloys book". ASM specialty handbook, ASM International. 1 ed., cap 1, pp. 3. Ohio, USA. ISBN: 978-0-87170-496-2. 1993.

[2] G.E. Totten and D.S. Mackenzie. "Handbook of aluminum". Physical Metallurgy and Processes, Marcel Dekker Inc., $1^{\text {ra }}$ ed. Vol. 1, Cap. 2, pp. 33. New York, USA. ISBN: 0-8247-0494-0. 2003.

[3] J. Randolph Kissell and R.L. Ferry. "Aluminum Structures: A guide to their specifications and design". Jhon Wiley \& Sons, Inc., Cap. 3, pp. 31. New York, USA. ISBN: 0-471-019658. 2002.

[4] N. Scotchmer y K. Chan. "¿Qué hay de Nuevo para soldar aluminio en la industria automotriz?". Welding Journal en español. Vol. $5 \mathrm{~N}^{\circ}$ 2, pp.10-15. 2012. ISSN: 2155-5559.

[5] J.M. Fortain and P. Lefebre. "Process for the MIG welding of aluminum and its alloys with a shielded gas of the $\mathrm{Ar} / \mathrm{He} / \mathrm{O}_{2}$ type". Washington, DC: U.S. Patent and Trademark Office, U.S. Patent $N^{\circ}$ 6,586,700. United States. 2003.

[6] E.E. Niebles Núñez, J. Unfried-Silgado y J.E. Torres Salcedo. "Metodología para el estudio de soldabilidad en uniones soldadas". Información Tecnológica. Vol. $25 \mathrm{~N}^{\mathrm{o}} 1$, pp. 3-14. 2014. ISSN: 0718-0764 http://dx.doi. org/10.4067/S0718-07642014000100002

[7] W.J. Arbegast. "Friction stir welding after a decade of development". Welding Journal. Vol. $85 \mathrm{~N}^{\text {o }}$ 7, pp. 28-35. 2006. ISSN: 0043-2296.

[8] R. Nandan, T. DebRoy and H.K. Bhadeshia, "Recent advances in friction-stir welding Process, weldment structure and properties". Progress in Materials Science. Vol. $53 \mathrm{~N}^{\circ} 6$, pp. 980-1023. 2008. ISSN: 0079-6425. URL: http://doi.org/10.1016/j.pmatsci.2008.05.001 H. Fujii, M. Ling Cui and K. Maeda. "Effect of tool shape on mechanical properties and microstructure of friction stir welded aluminum alloys". Materials Science and Engineering: A. Vol. 419 No 1-2, pp. 25-31. ISSN: 0921-5093, 2006. URL: http://doi. org/10.1016/j.msea.2005.11.045

[10] J. Zapata, J. Valderrama, E. Hoyos and D. López. "Mechanical properties comparison of friction stir welding butt joints of AA1100 made in a conventional milling machine and a FSW machine". Dyna. Vol. $80 \mathrm{~N}^{\circ} 182$, pp. 115-123. ISSN: 0012-7353. 2013.

[11] P.A. Schweitzer. "Metallic Materials Physical, Mechanical, and Corrosion Properties". Marcel Dekker, Inc, CRC Press. Cap. 19. New York, USA. ISBN: 0-8247-0878-4. 2003.

[12] E. Otero. "Aleaciones resistentes a la corrosión". In Corrosión y Degradación de Materiales. Síntesis editorial, Vallehermoso - España, pp. 309. ISBN: 84-7738-518-1. 2011.

[13] H. Ezuber, A. El-Houd and F. El-Shawesh. "A study on the corrosion behavior of aluminum alloys in seawater". Materials and Design. Vol. 29, pp. 801-805. 2008. ISSN: 0264-1275. URL: http://doi.org/10.1016/j. matdes.2007.01.021

[14] J. Soltis, N.J. Laycock and D. Krouse. "Temperature dependence of the pitting potential of high purity aluminum in chloride containing solution". Corrosion Science. Vol. $53 \mathrm{~N}^{\circ}$ 1, pp. 7-10. 2010. ISSN: 0010-938X. URL: http://doi.org/10.1016/j. corsci.2010.09.046

[15] S. Rajakumar and V. Balasubramanian. "Multi-Response Optimization of FrictionStir-Welded AA1100 Aluminum Alloy Joints". Journal of Materials Engineering and Performance. Vol. $21 \mathrm{~N}^{\mathrm{o}}$ 6, pp. 809-822. 2012. ISSN: 1059-9495. DOI: $10.1007 /$ s11665-011-9979-Z

[16] ASTM B117. "Standard Practice for Operating Salt Spray (fog) apparatus". STM International, West Conshohocken, PA. 2011. URL: www.astm.org

[17] ASTM G1-03. "Standard Practice for Preparing, Cleaning, and Evaluating Corrosion Test Specimens". ASTM International, West Conshohocken, PA, 2011. URL: www.astm. org DOI: 10.1520/G0001-03R11

[18] ASTM G5-14. Standard Reference Test Method for Making Potentiodynamic Anodic Polarization Measurements. ASTM 
International, West Conshohocken, PA. 2014. www.astm.org. DOI: 10.1520/G0005-14

[19] J. Unfried-Silgado, A. Torres and J.C. Carrasco Rodríguez. "Effects of shoulder geometry of tool on microstructure and mechanical properties of friction stir welded joints of AA1100 aluminum alloy". DYNA. Vol. 84 No 200, pp. 202-208. ISSN: 2346-2183.
2017. URL: https://doi.org/10.15446/dyna. v84n200.55787

[20] C.S. Paglia and R.G. Buchheit. "A look in the corrosion of aluminum alloy friction stir welds". Scripta Materialia. Vol. 58 No 5, pp. 383-387. ISSN: 1359-6462. 2008. URL: http://doi.org/10.1016/j.scriptamat. 2007.10.043 